\title{
Physical characteristic measurements for the neutron beam generated by the linac-based neutron source for BNCT in University of Tsukuba
}

\author{
Hiroaki Kumada ${ }^{1)}$, Kenta Takada2), Susumu Tanaka1), Yoshitaka Matsumoto1), Fujio Naito3),

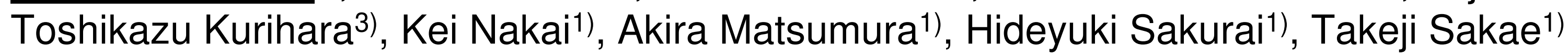

1) University of Tsukuba

2) Gunma Prefectural College of Health Sciences

3) High Energy Accelerator Research Organization

[Introduction] The University of Tsukuba is being developed a linac-base neutron source (iBNCT) for boron neutron capture therapy (BNCT). The device had been completed to produce and we have carried out the commissioning and conditioning to generate neutron beam with the high current proton beam. Fig.1(a) shows RFQ \& DTL type linac of the iBNCT neutron source device. And Fig.1-(b) shows the irradiation room of the iBNCT facility. At present, various characteristic measurements have been performed to confirm the practicability and applicability of the neutron beam to actual clinical trials using the device.

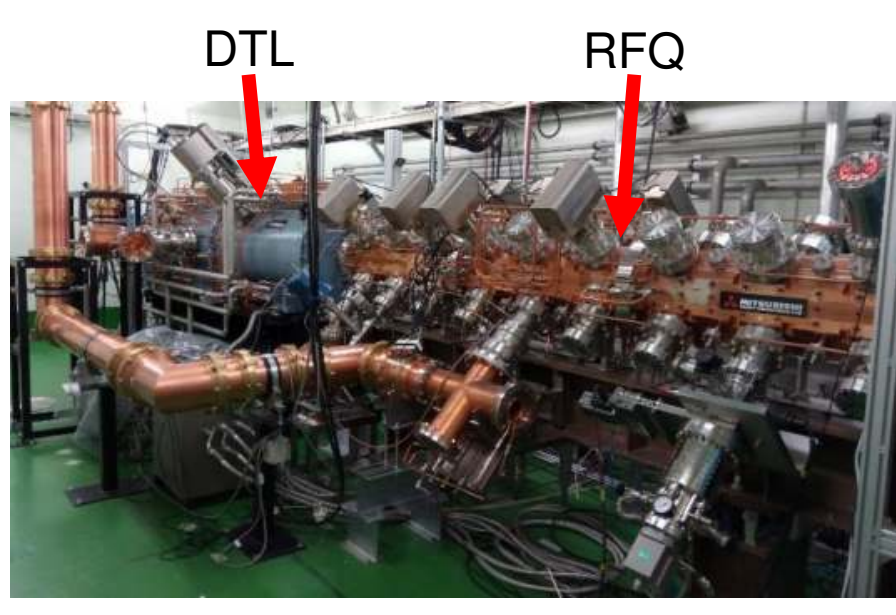

(a)

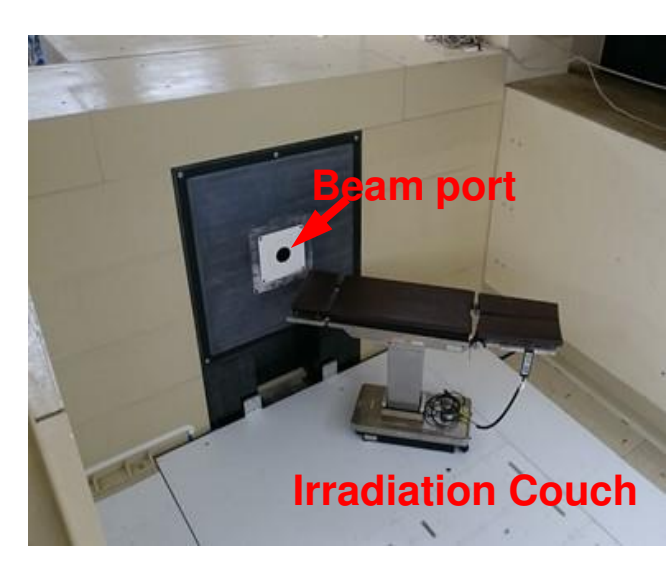

(b)
Fig.1 RFQ and DTL type linac (a) and irradiation room (b)

[Materials and methods] Various neutron irradiation experiments with a rectangular water phantom were performed. (1) For estimation of the two-dimensional distributions for thermal neutron flux and gamma-ray dose in human body, a neutron beam irradiation experiments using a water phantom were performed. For the thermal neutron flux measurement, gold wires were set inside the phantom. And for gamma-ray dose rate, many TLDs were also set in the phantom. Fig.2 shows schema of the phantom experiments for thermal neutron flux measurement. In the experiments, average proton beam current was set to $1.4 \mathrm{~mA}$, neutrons have been generated.

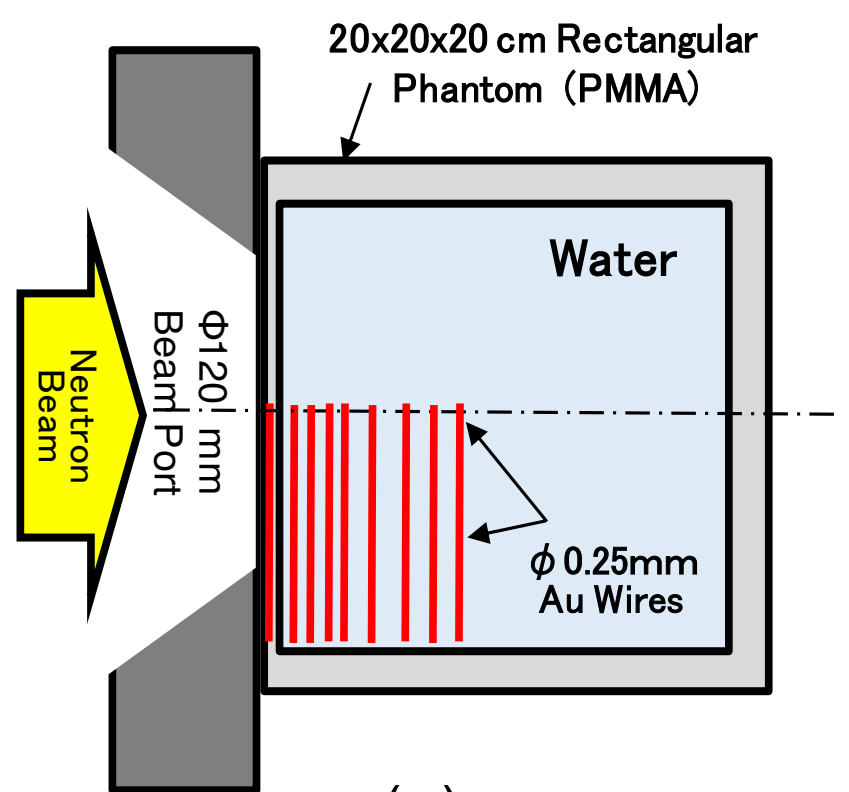

(a)

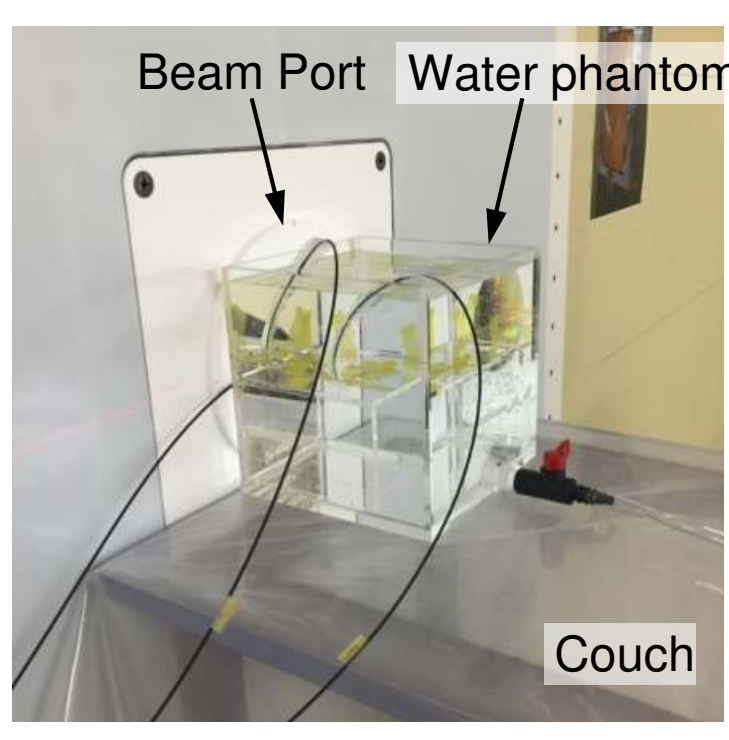

(b)
Fig.2 Schema of the water phantom with gold wires (a), and a picture for the phantom experiment (b)
(2) Stability test of the Be target has been performed. Some thermal neutron fluxes in the phantom were compared.

[Results and discussions] For the experimental results of the water phantom irradiation, the maximum values for thermal neutron flux and for gamma-ray dose rate in $1.4 \mathrm{~mA}$ operation were approximately $7.3 \times 10^{8}\left(\mathrm{n} / \mathrm{cm}^{2} \mathrm{~s}\right)$ and $2.7(\mathrm{~Gy} / \mathrm{h})$ at $2 \mathrm{~cm}$ depth in the phantom, respectively. Fig. 3 shows the distribution of the thermal neutron flux on the beam axis in the phantom.

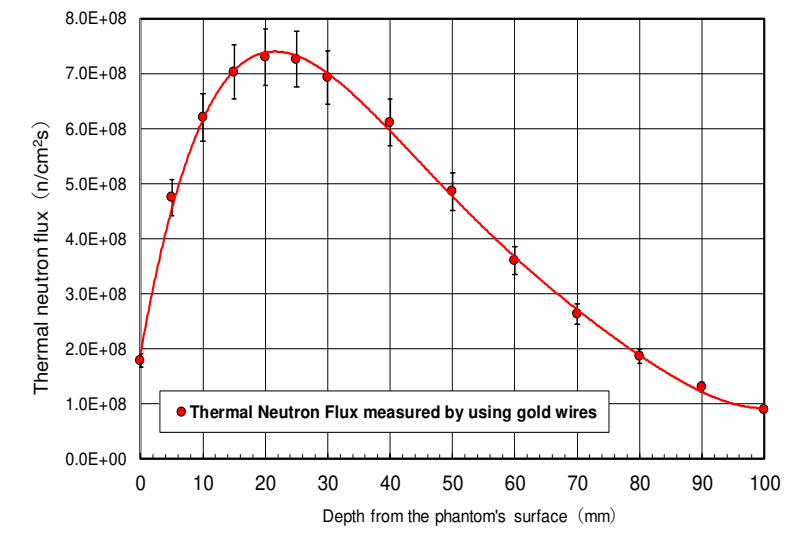

(a)

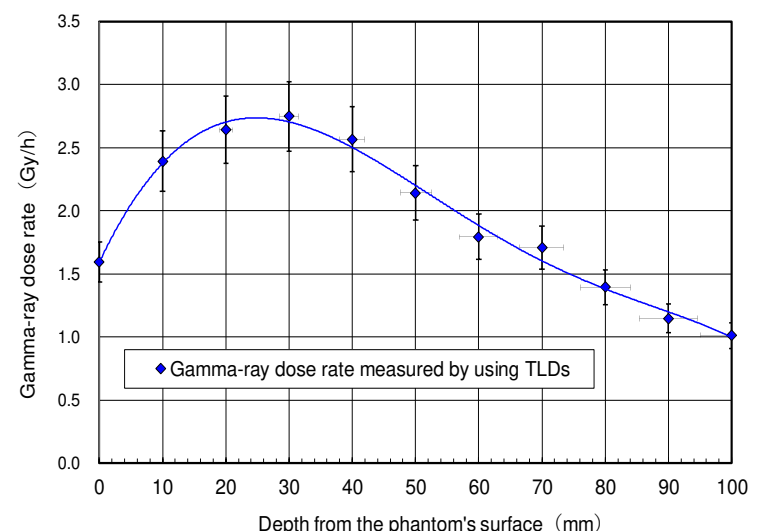

(b)
Fig.3 Distributions for thermal neutron flux (a) and gamma-ray dose rate (b) on the beam axis in the phantom

The beryllium target had received over 2,000-coulomb proton beams until now. This received proton amount is comparable to the amount that can emit neutrons that can treat more than 500 patients. Fig. 4 shows the proton charged quantity to Be target, and the thermal neutron flux values for each evaluated point in the phantom. The values were not decreased within error margins. The results demonstrated that the Be target is not deteriorated due to the neutron intensity is constant.

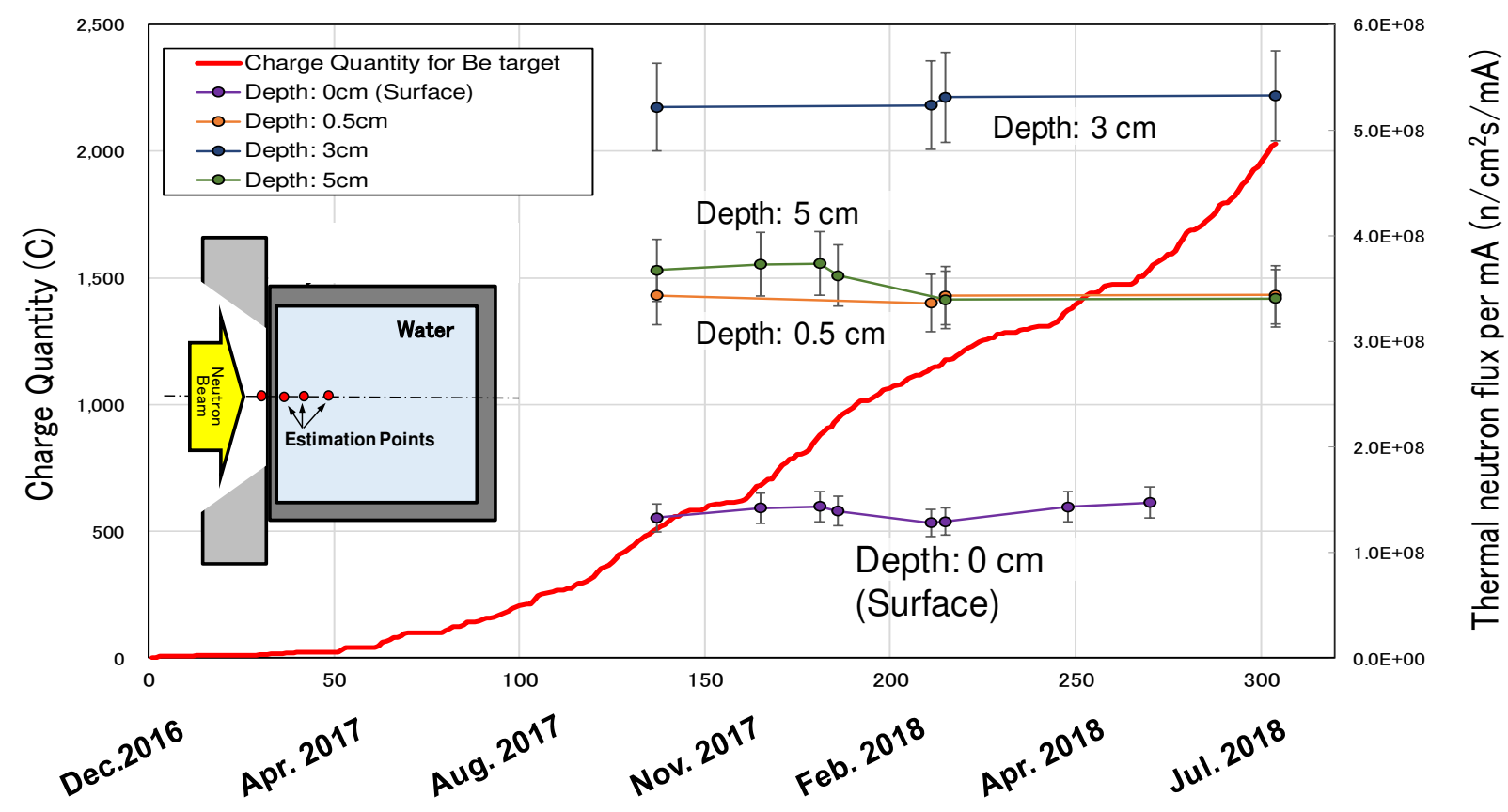

Fig.4 Charge quantity of proton beam to Be target and the measured thermal neutron flux at each depth in the phantom.

[Conclusions] At present, the proton current has been increased to $2.8 \mathrm{~mA}$. And non-clinical studies are performed within 2019, finally, we wish studies. to implement actual clinical study based on the results of the non-clinical 